\title{
The Association between Serum Uric Acid and Appendicular Skeletal Muscle Mass and the Effect of Their Interaction on Mortality in Patients on Peritoneal Dialysis
}

\author{
Xi Xiao ${ }^{a, b}$ Chunyan Yia,b Yuan Peng ${ }^{a, b}$ Hongjian Ye ${ }^{a, b}$ Haishan $W^{a, b}$ \\ Meiju $\mathrm{Wu}^{\mathrm{a}, \mathrm{b}}$ Xuan Huang ${ }^{\mathrm{a}, \mathrm{b}}$ Xueqing $\mathrm{Yu}^{\mathrm{a}, \mathrm{b}} \quad$ Xiao Yang ${ }^{\mathrm{a}, \mathrm{b}}$ \\ aDepartment of Nephrology, The First Affiliated Hospital, Sun Yat-sen University, \\ Guangzhou, China; ${ }^{b}$ Key Laboratory of Nephrology, Committee of Health and Guangdong \\ Province, Guangzhou, China
}

\section{Keywords}

Peritoneal dialysis · Serum uric acid · Appendicular skeletal muscle mass · Mortality

\begin{abstract}
Background: Serum uric acid (SUA) has been revealed to be positively associated with the body composition parameters in hemodialysis patients, but few studies have investigated that in patients on peritoneal dialysis (PD). The aim of this study was to identify the relationship between SUA and appendicular skeletal muscle mass (ASM) and the effect of their interaction on mortality in PD patients. Methods: This was a single-center retrospective cohort study. Patients who underwent multifrequency bioelectrical impedance analysis between January 1 , 2013, and December 31, 2016, and had data on SUA values were enrolled. All patients were followed up until December 31, 2019. Results: In total, 802 prevalent PD patients (57.9\% male), with mean age of $46.2 \pm 14.2$ years were enrolled. The average SUA and ASM were $6.8 \pm 1.3$ $\mathrm{mg} / \mathrm{dL}$ and $21.2 \pm 4.9 \mathrm{~kg}$. According to multiple linear regression models, SUA was positively associated with relative ASM in middle-aged and older PD patients (standardized coefficients [ $\beta$ ] 0.117; 95\% confidence interval [Cl] 0.027, 0.200; $p=0.010$ ). Further sex-stratified analysis showed that the association existed only in males $(\beta 0.161 ; 95 \% \mathrm{Cl} 0.017,0.227 ; p=0.023)$. Moreover, the presence of hyperuricemia was found to predict lower risk of all-cause mortality (hazard ratio $[\mathrm{HR}] 0.514,95 \% \mathrm{Cl} 0.272,0.970 ; p=0.040$ ) only in patients with lower relative ASM. And, the adjusted HR of every $1 \mathrm{mg} / \mathrm{dL}$ elevated SUA level was $0.770(95 \% \mathrm{Cl} 0.609,0.972$; $p=0.028)$ for all-cause mortality in the lower relative ASM subgroup. Conclusions: There exists a positive association between the SUA and ASM, and the ASM significantly affected the association between SUA and all-cause PD mortality.




\section{Introduction}

Uric acid (UA), the end product of purine metabolism, has gained increasing attention over conflicting reports on its effects. High serum uric acid (SUA) was reported to be associated with higher mortality in individuals at high risk for cardiovascular disease (CVD) but has no independently predictive value in low-risk individuals [1,2]. Given that SUA is excreted primarily by the kidneys, hyperuricemia is common in patients undergoing peritoneal dialysis (PD), who are believed to have a higher risk of CVD and mortality compared with the general population [3]. However, there are discrepant reports on the effects of SUA on prognosis in PD patients. While some trials showed that higher SUA is associated with higher mortality in PD patients, others demonstrated no such association [4]. Furthermore, a recent study reported the opposite effect whereby higher SUA levels were associated with a lower risk of all-cause and CVD mortality in female PD patients [5]. Another study analyzing 309 PD patients revealed that decline of SUA during the follow-up period may predict all-cause mortality [6]. These contradictory results may partially be explained by the numerous interactions between SUA and other risk factors that contribute to mortality in PD patients [4, 7]. Therefore, it remains to be determined if SUA is independently associated with PD mortality. Despite its uncertain effects, SUA has been identified as a nutritional marker in hemodialysis (HD) patients and was shown to correlate positively with nutritional markers and body composition parameters [8]. As a common feature of protein-energy wasting [9], lower appendicular skeletal muscle mass (ASM) was shown to independently predict mortality in PD patients $[10,11]$. The gender and diabetics status were revealed to modify the prognostic effect, and the study by Xiang et al. [12] suggested that the adverse effect of higher SUA level on all-cause mortality was also more prominent in hypoalbuminemia and normal-weight groups of PD patients. However, the relationship between SUA and ASM was unknown in PD population and the effect of the ASM level on the association between SUA and all-cause or CVD mortality in PD patients remains to be explored. Therefore, in the present study, we aimed to investigate the association between SUA and ASM and determine if their potential interactions are related to PD mortality. Because of sex-related differences in these 2 parameters, we performed subgroup analyses to determine if the association between SUA and ASM is different in males and females. Additionally, given that the decline in ASM accelerates with age [13], and previous studies have focused primarily on middle-aged and older populations $[14,15]$, additional subgroup analyses were performed to determine if the association differed between younger and older PD populations.

\section{Materials and Methods}

\section{Patients}

In this study, continuous ambulatory peritoneal dialysis patients who underwent multifrequency bioelectrical impedance analysis (BIA) between January 1, 2013, and December 31,2016 , in our PD center were recruited. The inclusion criteria were as follows: prevalent continuous ambulatory peritoneal dialysis, age $>18$-year-olds, and underwent PD at least 3 months from the time of BIA. Patients, who were transferred to the PD center following a failed renal transplantation, were undergoing long-term HD ( $>3$ months), had cancer, or lacked data on SUA within 3 months from BIA were excluded.

\section{Data Collection}

We collected data on demographic and clinical parameters and BIA. CVD was defined as history of congestive heart failure, angina, myocardial infarction, cerebrovascular disease, or

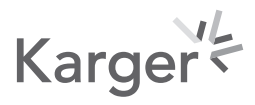


peripheral vascular disease [16]. Charlson score [17] was used to assess comorbidity. Total $\mathrm{Kt} / \mathrm{V}$ (measured according to dialysate effluent, 24-h urine output, and blood analysis of serum urea nitrogen) and residual kidney function indexed by the glomerular filtration rate (measured according to 24-h urine samples and adjusted for body surface area) were obtained using PD Adequest software 2.0 (Baxter, Deerfield, IL, USA). Biochemical parameters of PD patients were assessed with dialysate in their abdominal space in the morning, in the Department of Clinical Laboratory in our hospital according to standard protocols. Body composition parameters were measured using BIA with an InBody 720 instrument (Biospace, Seoul, Korea) as previously described [18]. The BIA measurements were performed with a peritoneal solution in the abdominal space since it has been previously shown no significant effect on BIA measures. All of the subjects were guided to fast and avoid exercise for $8 \mathrm{~h}$ prior to measurement and rest at least $30 \mathrm{~min}$ before the measurement. According to the manufacturer's instructions, the subjects were in the upright position, without shoes or extra clothing, and stood on 4-foot electrodes: 2 oval electrodes and 2 heel-shaped electrodes. They were also guided to grab 2 palm and thumb electrodes to get the thumb and palm electrodes. The skin and electrodes were pre-cleaned using specific electrolyte tissue as instructed. The visceral fat area was calculated according to the regression formula of body fat percentage, trunk impedance, and lower limb muscle mass with the visceral fat area in the cross-sectional area of 4-5 lumbar spines by means of the gold standard of CT. The ratio of extracellular water to total body water over 0.40 was identified as overhydration status [18]. The left and right upper limb muscle mass and left and right lower limb muscle mass were provided by the BIA instrument, and the ASM was defined as the sum of the muscle mass of the limbs. The relative ASM of appendicular skeletal muscle index (ASMI), computed as ASM $/$ height $^{2}\left(\mathrm{~kg} / \mathrm{m}^{2}\right.$ ), which was recommended as the diagnostic index for sarcopenia by the Asian Working Group for Sarcopenia [19], was used as an indicator of ASM for the effect of body size. Data on medication history were obtained from the medical records of patients in our PD center. Agegroups (based on definitions in the MEDLINE database) were as follows: young (19-44 years), middle-aged (45-64 years), and older (65 years and over) [20]. The presence of hyperuricemia was defined as SUA $>7 \mathrm{mg} / \mathrm{dL}(420 \mu \mathrm{mol} / \mathrm{L})$ or taking UA-lowering agents [21]. The primary outcome of this study was all-cause mortality. The second outcome was CVD mortality, which included death from cardiovascular events, including myocardial infarction, congestive heart failure, cardiac arrest, cardiac arrhythmia, or cerebrovascular accident.

\section{Statistical Analysis}

Patients were grouped into tertiles according to SUA values. Data are presented as mean \pm SD for normally distributed quantitative variables, median and interquartile range for abnormal quantitative variables, and frequency and percentage for categorical variables. Comparisons of the characteristics of PD patients in different SUA tertiles were performed using a line trend test by modeling the ordered SUA tertiles as a 1 degree-of-freedom linear variable in the linear regression model. Correlation analysis of the SUA with other variables was performed using the Pearson correlation test for normally distributed variables or Spearman rank correlation test for nonparametric variables, and the multiple tests were adjusted by the false-discovery rate of the Benjamini-Hochberg method. The association between SUA and the ASMI was tested using multiple linear regression models. Covariables that demonstrated significant linear trends in different SUA tertiles with $p<0.05$ in the univariate analysis or that possessed clinical significance were selected for the full model. Given that previous studies reported the effects of 25 -hydroxyvitamin D on skeletal muscle mass [22], the use of active vitamin D3 in PD patients was forced to enter the full model. Allowing for differences in SUA and ASM according to sex and age, subgroup analyses were performed to further explore whether there are sex- or age-specific relationships involving

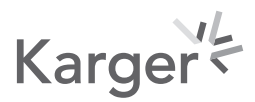


Patients performed BIA at our PD center from January 1 , 2013 to December 31, $2016(\mathrm{n}=1000)$

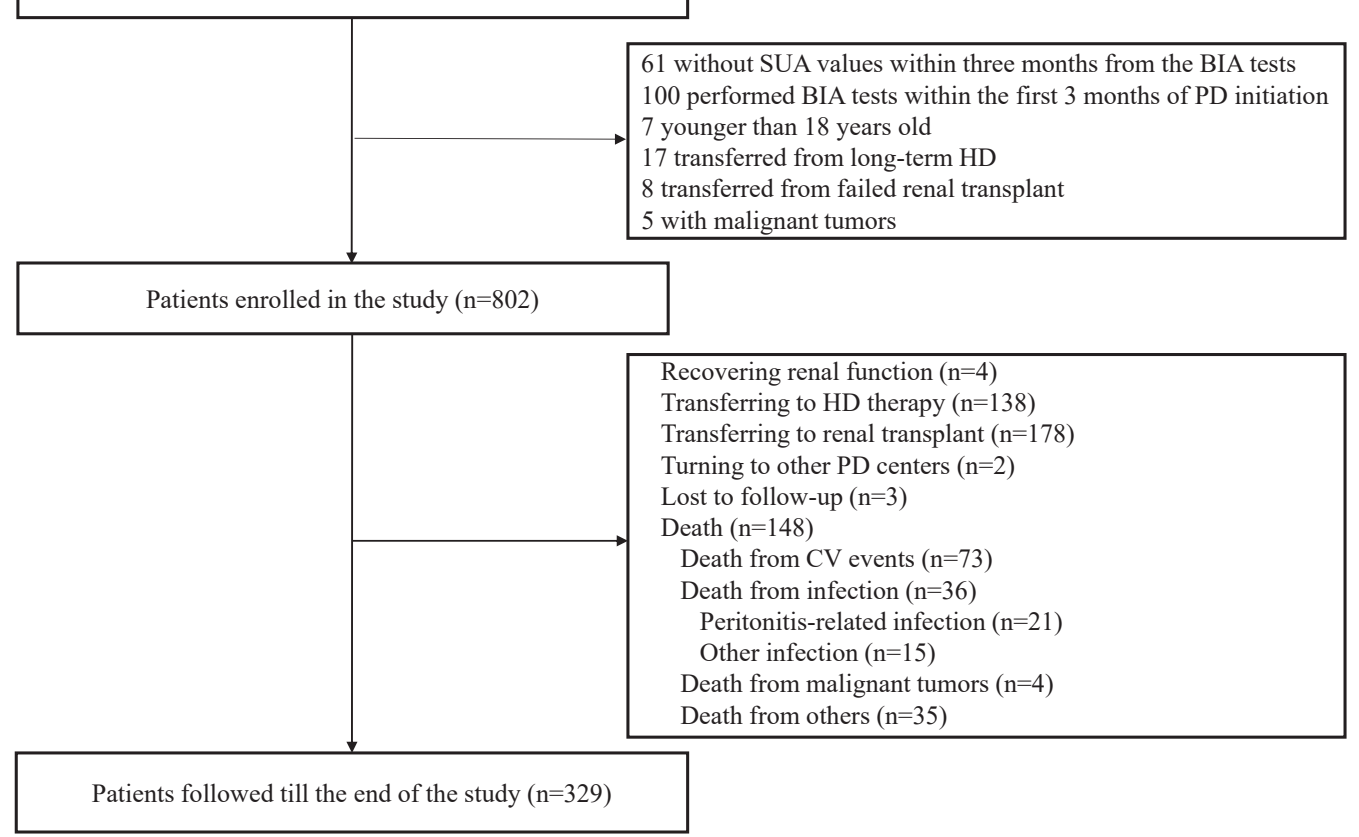

Fig. 1. The flowchart for the recruitment process and outcome of PD patients in the study. BIA, bioelectrical impedance analysis; CV, cardiovascular; HD, hemodialysis; PD, peritoneal dialysis; SUA, serum uric acid.

these parameters in PD patients. The Cox proportional-hazard model was used to evaluate the effects of SUA on all-cause and CVD mortality in PD patients. The time at risk in this study was from PD catheterization to death, renal function recovery, transfer to long-term HD, renal transplantation, transfer to another PD center, loss to follow-up, or to the time point of December 31, 2019. All statistical analyses were performed using SPSS statistical software (version 20.0). Statistical significance was set as a two-sided $p$ value $<0.05$.

\section{Results}

\section{Patient Characteristics}

The process of patient recruitment is shown in Figure 1. A total of 802 patients $(57.9 \%$ male) were enrolled, with mean age of $46.2 \pm 14$.2 years and median PD vintage of 18.9 (6.641.0) months. The primary kidney diseases were chronic glomerulonephritis (66.1\%), diabetic nephropathy (14.8\%), and hypertensive lesions (8.2\%). Patients had mean ASM of $21.2 \pm 4.9 \mathrm{~kg}$ and mean SUA of $6.8 \pm 1.3 \mathrm{mg} / \mathrm{dL}$. Compared with participants in the lowest SUA tertile, the higher and highest tertiles showed greater ASMI $(7.7 \pm 1.4$ vs. $7.8 \pm 1.3,8.0 \pm 1.4$ $\mathrm{kg} / \mathrm{m}^{2}$ ). As shown in Table 1, patients in the higher SUA tertile were more likely to be male and less likely to use diuretics. Moreover, they had higher neutrophil to lymphocyte ratio (N/L), serum albumin, phosphorus, triglyceride, urea nitrogen, creatinine, intact parathyroid hormone (iPTH), ASM, waist and arm circumference, visceral fat area, fat mass, and BMI, and lower total Kt/V and corrected calcium.

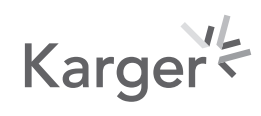


Table 1. Characteristics of the PD patients by tertiles of SUA

\begin{tabular}{|c|c|c|c|c|c|}
\hline Variables & $\begin{array}{l}\text { Total, } \\
n=802\end{array}$ & $\begin{array}{l}\text { Tertile } 1 \\
\text { (267) }\end{array}$ & $\begin{array}{l}\text { Tertile } 2 \\
(267)\end{array}$ & $\begin{array}{l}\text { Tertile } 3 \\
\text { (268) }\end{array}$ & $\begin{array}{l}p \text { value } \\
\text { for trend }\end{array}$ \\
\hline SUA, mg/dL & $6.8 \pm 1.3$ & $5.5 \pm 0.7$ & $6.8 \pm 0.3$ & $8.2 \pm 0.9$ & - \\
\hline Age, years & $46.2 \pm 14.2$ & $46.4 \pm 14.4$ & $46.1 \pm 14.3$ & $46.2 \pm 13.9$ & 0.819 \\
\hline Male, $n(\%)$ & $464(57.9)$ & $133(49.8)$ & $155(58.1)$ & $176(65.7)$ & $<0.001$ \\
\hline Diabetes, $n(\%)$ & $147(18.3)$ & 47 (17.6) & $48(18.0)$ & $52(19.4)$ & 0.591 \\
\hline CVD, $n(\%)$ & $146(18.2)$ & $41(15.4)$ & $57(21.3)$ & $48(17.9)$ & 0.446 \\
\hline Charlson comorbidity score & $3(2-4)$ & $3(2-4)$ & $3(2-4)$ & $3(2-4)$ & 0.971 \\
\hline Chronic glomerulonephritis, $n(\%)$ & $530(66.1)$ & $181(67.8)$ & $177(66.3)$ & $172(64.2)$ & 0.378 \\
\hline Diabetic nephropathy, $n(\%)$ & $119(14.8)$ & $38(14.2)$ & $41(15.4)$ & 40 (14.9) & 0.822 \\
\hline Hypertensive kidney lesion, $n(\%)$ & $66(8.2)$ & $22(8.2)$ & $20(7.5)$ & $24(9.0)$ & 0.763 \\
\hline PD vintage, months & $18.9(6.6-41.0)$ & $18.9(6.6-41.5)$ & $20.9(6.9-42.4)$ & $17.2(6.1-40.7)$ & 0.223 \\
\hline $\mathrm{Kt} / \mathrm{V}$ & $2.4 \pm 0.8$ & $2.4 \pm 0.9$ & $2.4 \pm 0.8$ & $2.2 \pm 0.7$ & 0.005 \\
\hline Residual urine volume, mL/day & $500(150-1,000)$ & $500(100-1,000)$ & $500(162.5-1,000)$ & $550(200-1,000)$ & 0.760 \\
\hline Systolic pressure, mm Hg & $137.3 \pm 20.4$ & $137.3 \pm 19.8$ & $137.9 \pm 20.0$ & $136.8 \pm 21.4$ & 0.802 \\
\hline Diastolic pressure, $\mathrm{mm} \mathrm{Hg}$ & $84.4 \pm 13.4$ & $84.4 \pm 13.2$ & $84.5 \pm 13.9$ & $84.3 \pm 13.1$ & 0.963 \\
\hline Hemoglobin, g/dL & $11.2 \pm 2.0$ & $11.1 \pm 1.9$ & $11.2 \pm 1.9$ & $11.2 \pm 2.2$ & 0.406 \\
\hline $\mathrm{N} / \mathrm{L}$ & $3.1(2.4-4.2)$ & $3.1(2.2-3.9)$ & $3.2(2.5 \pm 4.2)$ & $3.3(2.4 \pm 4.3)$ & 0.011 \\
\hline $\mathrm{ALP}, \mathrm{U} / \mathrm{L}$ & $76.0(59.0-100.0)$ & $73.0(56.8-99.3)$ & $76.5(59.0-97.5)$ & $77.0(61.0-100.8)$ & 0.061 \\
\hline Serum albumin, g/dL & $3.8 \pm 0.4$ & $3.7 \pm 0.4$ & $3.8 \pm 0.5$ & $3.8 \pm 0.4$ & 0.001 \\
\hline Serum prealbumin, mg/L & $376(325-435)$ & $383(323-433)$ & $377(333-440)$ & $373(322-432)$ & 0.541 \\
\hline Corrected calcium, mg/dL & $9.4 \pm 0.7$ & $9.5 \pm 0.6$ & $9.4 \pm 0.7$ & $9.3 \pm 0.8$ & 0.004 \\
\hline Serum phosphorus, mg/dL & $5.1 \pm 1.8$ & $4.5 \pm 1.3$ & $5.2 \pm 1.4$ & $5.7 \pm 2.2$ & $<0.001$ \\
\hline Total cholesterol, mg/dL & $193.3(162.4-224.2)$ & $189.4(161.4-228.1)$ & $185.6(162.4-220.4)$ & $197.2(166.2-232.0)$ & 0.225 \\
\hline Triglyceride, mg/dL & $126.7(90.4-183.0)$ & $117.0(85.7-172.8)$ & $125.8(89.5-182.5)$ & $138.2(100.3-188.7)$ & 0.001 \\
\hline HDL-C, mg/dL & $43.0(35.4-52.6)$ & $45.0(37.1-54.9)$ & $42.9(35.2-50.6)$ & $41.0(34.0-52.4)$ & 0.974 \\
\hline $\mathrm{LDL}-\mathrm{C}, \mathrm{mg} / \mathrm{dL}$ & $112.5(90.1-141.4)$ & $112.9(88.7-141.0)$ & $110.2(89.7-135.7)$ & $113.7(90.9-143.4)$ & 0.861 \\
\hline Serum urea nitrogen, $\mathrm{mg} / \mathrm{dL}$ & $50.7(40.8-61.6)$ & $45.6(36.7-54.3)$ & $51.0(40.9-61.3)$ & $57.1(48.0-67.6)$ & $<0.001$ \\
\hline Serum $\mathrm{Cr}, \mathrm{mg} / \mathrm{dL}$ & $10.8(8.4-13.5)$ & $10.1(8.1-12.5)$ & $11.0(8.0-13.8)$ & $11.7(8.6-14.5)$ & $<0.001$ \\
\hline $\mathrm{iPTH}, \mathrm{pg} / \mathrm{mL}$ & $344.4(182.8-617.3)$ & $299.0(174.3-535.1)$ & $331.6(177.8-575.5)$ & $397.8(212.0-792.5)$ & 0.001 \\
\hline Leg muscle mass, kg & $16.2 \pm 3.8$ & $15.8 \pm 3.9$ & $16.1 \pm 3.6$ & $16.6 \pm 3.9$ & 0.008 \\
\hline Arm muscle mass, $\mathrm{kg}$ & $5.0 \pm 1.4$ & $4.7 \pm 1.3$ & $5.0 \pm 1.3$ & $5.3 \pm 1.4$ & $<0.001$ \\
\hline ASM, kg & $21.2 \pm 4.9$ & $20.5 \pm 5.0$ & $21.1 \pm 4.7$ & $22.0 \pm 5.0$ & 0.001 \\
\hline Waist circumference, $\mathrm{cm}$ & $79.3 \pm 10.2$ & $77.9 \pm 9.9$ & $78.6 \pm 10.4$ & $81.3 \pm 10.0$ & $<0.001$ \\
\hline Arm circumference, $\mathrm{cm}$ & $28.6 \pm 3.2$ & $27.9 \pm 3.0$ & $28.5 \pm 3.1$ & $29.5 \pm 3.2$ & $<0.001$ \\
\hline Visceral fat area, $\mathrm{cm}^{2}$ & $38.1(5.0-73.2)$ & $36.1(5.0-67.9)$ & $30.1(5.0-70.4)$ & $49.5(10.9-78.8)$ & 0.008 \\
\hline Fat mass, kg & $12.3 \pm 6.8$ & $11.8 \pm 6.5$ & $11.9 \pm 7.0$ & $13.3 \pm 6.9$ & 0.008 \\
\hline ECW/TBW & $0.40(0.39-0.41)$ & $0.40(0.39-0.41)$ & $0.40(0.39-0.41)$ & $0.40(0.39-0.40)$ & 0.016 \\
\hline $\mathrm{BMI}, \mathrm{kg} / \mathrm{m}^{2}$ & $23.2 \pm 3.4$ & $22.8 \pm 3.2$ & $23.0 \pm 3.4$ & $23.8 \pm 3.5$ & $<0.001$ \\
\hline ACEI and/or ARB use, $n(\%)$ & 567 (70.7) & $195(73.0)$ & $187(70.0)$ & $185(69.0)$ & 0.310 \\
\hline Diuretic use, $n(\%)$ & $90(11.2)$ & $41(15.4)$ & $24(9.0)$ & $25(9.3)$ & 0.027 \\
\hline Active vitamin $\mathrm{D}_{3}$ use, $n(\%)$ & $397(49.5)$ & $144(53.9)$ & $125(46.8)$ & $128(47.8)$ & 0.154 \\
\hline UA-lowering agent use, $n(\%)$ & $85(10.6)$ & $31(11.6)$ & $29(10.9)$ & $25(9.3)$ & 0.392 \\
\hline
\end{tabular}

Values are presented as means \pm SD or interquartile range or percentages. ACEI, angiotensin-converting enzyme inhibitor; ALP, alkaline phosphatase; ARB, angiotensin II receptor blocker; ASM, appendicular skeletal muscle mass; CVD, cardiovascular disease; ECW, extracellular water; HDL-C, high-density lipoprotein cholesterol; iPTH, intact parathyroid hormone; LDL-C, low-density lipoprotein; N/L, neutrophil to lymphocyte ratio; PD, peritoneal dialysis; SUA, serum uric acid; TBW, total body water; UA, uric acid. ${ }^{a} p$ value for line trend between the SUA tertiles.

\section{Association of SUA and ASMI}

As shown in Table 2, the serum urea nitrogen, phosphorus, arm circumference, serum creatinine, albumin, corrected calcium, ASMI, iPTH, triglyceride, BMI, total Kt/V, waist circumference, high-density lipoprotein cholesterol, visceral fat area, and N/L were significantly correlated with SUA in PD patients (the scatter plots between SUA and the abovementioned variables were shown in online supplementary Fig. 1-4; for all online supplementary material, 
Kidney and

Blood Pressure Research

Table 2. Correlation analysis between SUA and other parameters in PD patients

\begin{tabular}{|c|c|c|c|c|}
\hline \multicolumn{5}{|c|}{ Kidney Blood Press Res 2020;45:969-981 } \\
\hline DOI: $10.1159 / 000510746$ & \multicolumn{4}{|c|}{$\begin{array}{l}\text { (c) } 2020 \text { The Author(s). Published by S. Karger AG, Basel } \\
\text { www.karger.com/kbr }\end{array}$} \\
\hline \multicolumn{5}{|c|}{$\begin{array}{l}\text { Xiao et al.: Association and Interaction between Uric Acid and Appendicular Skeletal } \\
\text { Muscle Mass }\end{array}$} \\
\hline Variables & & $r$ & $p$ value & $\begin{array}{l}\text { FDR-adjusted } \\
p \text { value }\end{array}$ \\
\hline \multicolumn{2}{|c|}{ Serum urea nitrogen $(\mathrm{mg} / \mathrm{dL})$} & 0.356 & $<0.001$ & $<0.001$ \\
\hline \multicolumn{2}{|c|}{ Serum phosphorus (mg/dL) } & 0.289 & $<0.001$ & $<0.001$ \\
\hline \multicolumn{2}{|c|}{ Arm circumference $(\mathrm{cm})$} & 0.202 & $<0.001$ & $<0.001$ \\
\hline \multicolumn{2}{|c|}{ Serum $\operatorname{Cr}(\mathrm{mg} / \mathrm{dL})$} & 0.130 & $<0.001$ & 0.001 \\
\hline \multicolumn{2}{|c|}{ Serum albumin $(\mathrm{g} / \mathrm{dL})$} & 0.126 & $<0.001$ & 0.002 \\
\hline \multicolumn{2}{|c|}{ Corrected calcium (mg/dL) } & -0.120 & 0.001 & 0.004 \\
\hline \multicolumn{2}{|c|}{ ASMI $\left(\mathrm{kg} / \mathrm{m}^{2}\right)$} & 0.112 & 0.002 & 0.006 \\
\hline \multicolumn{2}{|l|}{ iPTH $(\mathrm{pg} / \mathrm{mL})$} & 0.108 & 0.003 & 0.008 \\
\hline \multicolumn{2}{|l|}{ Triglyceride (mg/dL) } & 0.107 & 0.003 & 0.008 \\
\hline \multicolumn{2}{|l|}{ BMI $\left(\mathrm{kg} / \mathrm{m}^{2}\right)$} & 0.106 & 0.003 & 0.008 \\
\hline \multicolumn{2}{|c|}{$\mathrm{Kt} / \mathrm{V}$} & -0.097 & 0.009 & 0.018 \\
\hline \multicolumn{2}{|c|}{ Waist circumference $(\mathrm{cm})$} & 0.095 & 0.007 & 0.017 \\
\hline \multicolumn{2}{|c|}{$\mathrm{HDL}-\mathrm{C}(\mathrm{mg} / \mathrm{dL})$} & -0.094 & 0.009 & 0.018 \\
\hline \multicolumn{2}{|l|}{ Visceral fat area $\left(\mathrm{cm}^{2}\right)$} & 0.086 & 0.015 & 0.028 \\
\hline \multicolumn{2}{|l|}{$\mathrm{N} / \mathrm{L}$} & 0.079 & 0.026 & 0.045 \\
\hline \multicolumn{2}{|l|}{ Fat mass (kg) } & 0.063 & 0.077 & 0.125 \\
\hline \multicolumn{2}{|c|}{$\operatorname{ALP}(\mathrm{U} / \mathrm{L})$} & 0.048 & 0.175 & 0.260 \\
\hline \multicolumn{2}{|c|}{ Total cholesterol (mg/dL) } & 0.048 & 0.180 & 0.260 \\
\hline \multicolumn{2}{|c|}{ PD vintage (months) } & -0.046 & 0.194 & 0.265 \\
\hline \multicolumn{2}{|l|}{ Age (years) } & -0.032 & 0.373 & 0.485 \\
\hline \multicolumn{2}{|c|}{ LDL-C (mg/dL) } & 0.025 & 0.491 & 0.581 \\
\hline \multicolumn{2}{|c|}{ Systolic pressure (mm Hg) } & 0.025 & 0.492 & 0.581 \\
\hline \multicolumn{2}{|c|}{ Charlson comorbidity score } & -0.022 & 0.525 & 0.593 \\
\hline \multicolumn{2}{|c|}{ Diastolic pressure (mm Hg) } & 0.021 & 0.563 & 0.610 \\
\hline \multicolumn{2}{|c|}{ Serum prealbumin $(\mathrm{mg} / \mathrm{L})$} & 0.019 & 0.603 & 0.627 \\
\hline \multicolumn{2}{|c|}{ Hemoglobin $(\mathrm{g} / \mathrm{dL})$} & 0.013 & 0.712 & 0.712 \\
\hline
\end{tabular}

ALP, alkaline phosphatase; ASMI, appendicular skeletal muscle mass index; FDR, false-discovery rate; HDL-C, high-density lipoprotein cholesterol; iPTH, intact parathyroid hormone; LDL-C, low-density lipoprotein; N/L, neutrophil to lymphocyte ratio; $\mathrm{PD}$, peritoneal dialysis; SUA, serum uric acid.

see www.karger.com/doi/10.1159/000510746). While no significant association was found between SUA and ASMI in total, male, or female PD patients in multiple linear regression models (shown in Table 3), given that a significant association between the interaction of the 2 age-groups (the young group and the middle-aged and older group) with SUA and ASMI was observed $(p=0.037)$, further age stratification analyses were performed which revealed a positive association in middle-aged and older PD patients (standardized coefficients [ $\beta]$ $0.117,95 \%$ confidence interval [CI] $0.027,0.200 ; p=0.010$ ), but not in young PD patients ( $\beta$ $-0.044,95 \% \mathrm{CI}-0.150,0.049 ; p=0.317$ ). After further stratification according to sex in middle-aged and older patients, SUA was found to be associated with ASMI in males ( $\beta 0.161$, $95 \%$ CI 0.017, 0.227; $p=0.023)$, but not in females ( $\beta 0.130,95 \%$ CI $-0.025,0.268 ; p=0.102)$. When the lowest SUA tertile was set as a reference group, elevated SUA in the higher tertile was found to be related to greater ASMI in males only ( $\beta$ 0.182, 95\% CI 0.040, 0.757; $p=$ 0.030) (Table 4). To further explore whether the sex-specific association was caused by higher SUA levels in male patients, a sensitivity analysis was performed. After excluding the highest SUA tertile in males and the lowest tertile in females, the significant association between SUA and ASMI was lost in males ( $\beta 0.112,95 \% \mathrm{CI}-0.085,0.353 ; p=0.227)$ and was still not decisively significant in females $(\beta 0.162,95 \% \mathrm{CI}-0.049,0.489 ; p=0.107)$ in the 
Table 3. Association of SUA with ASMI in total, male, female, young, middle-aged, and older patients, respectively

\begin{tabular}{|c|c|c|}
\hline Variables & $\beta(95 \% \mathrm{CI})$ & $p$ value $^{\mathrm{a}}$ \\
\hline Total patients $(n=802)$ & $0.042(-0.020,0.108)$ & 0.177 \\
\hline $\operatorname{Male}^{\mathrm{b}}(n=464)$ & $0.066(-0.028,0.139)$ & 0.192 \\
\hline Female $(n=338)$ & $0.043(-0.063,0.143)$ & 0.450 \\
\hline Middle-aged and older patients ${ }^{c}(n=408)$ & $0.117(0.027,0.200)$ & 0.010 \\
\hline Young patients $(n=394)$ & $-0.044(-0.150,0.049)$ & 0.317 \\
\hline
\end{tabular}

ASMI, appendicular skeletal muscle mass index; CI, confidence interval; PD, peritoneal dialysis; SUA, serum uric acid. a $p$ value of adjusting for age, gender, diabetes, CVD, PD vintage, total Kt/V, serum albumin, neutrophil to lymphocyte ratio, triglyceride, intact parathyroid hormone, use of active vitamin $\mathrm{D}_{3}$, uric acidlowering agent, and diuretic in the multiple linear regression models. ${ }^{\mathrm{b}}$ Did not adjust for sex or age when analyzing in the male and female PD patients or in the middle-aged, older, and young PD patients, respectively. ${ }^{c} p$ value of the association between the interaction of the 2 age groups, with SUA and ASMI, was 0.037, and the interaction between them was tested on the final model.

Table 4. Gender-stratified analysis of the association between SUA and ASMI in middle-aged and older PD patients

\begin{tabular}{|c|c|c|c|c|c|c|}
\hline \multirow[t]{2}{*}{ Variables } & \multicolumn{2}{|l|}{ Model $1^{\mathrm{a}}$} & \multicolumn{2}{|l|}{ Model $2^{b}$} & \multicolumn{2}{|l|}{ Model 3c } \\
\hline & $\beta(95 \% \mathrm{CI})$ & $p$ value & $\beta(95 \% \mathrm{CI})$ & $p$ value & $\beta(95 \% \mathrm{CI})$ & $p$ value \\
\hline \multicolumn{7}{|l|}{ Continuous SUA } \\
\hline Total & $0.097(0.015,0.172)$ & 0.020 & $0.093(0.008,0.171)$ & 0.031 & $0.110(0.022,0.191)$ & 0.013 \\
\hline Male & $0.136(0.008,0.196)$ & 0.034 & $0.116(-0.013,0.187)$ & 0.089 & $0.161(0.017,0.227)$ & 0.023 \\
\hline Female & $0.092(-0.055,0.227)$ & 0.232 & $0.109(-0.038,0.242)$ & 0.152 & $0.130(-0.025,0.268)$ & 0.102 \\
\hline \multicolumn{7}{|l|}{ Categorical SUA $^{\mathrm{d}}$} \\
\hline Total tertile 2 & $0.056(-0.106,0.408)$ & 0.248 & $0.052(-0.122,0.408)$ & 0.289 & $0.062(-0.100,0.437)$ & 0.219 \\
\hline Total tertile 3 & $0.112(0.046,0.560)$ & 0.021 & $0.105(0.019,0.543)$ & 0.035 & $0.123(0.059,0.601)$ & 0.017 \\
\hline Male tertile 2 & $0.032(-0.265,0.407)$ & 0.677 & $0.020(-0.310,0.401)$ & 0.800 & $0.090(-0.165,0.572)$ & 0.277 \\
\hline Male tertile 3 & $0.136(-0.030,0.630)$ & 0.075 & $0.122(-0.077,0.610)$ & 0.128 & $0.182(0.040,0.757)$ & 0.030 \\
\hline Female tertile 2 & $0.104(-0.155,0.646)$ & 0.227 & $0.115(-0.121,0.675)$ & 0.171 & $0.091(-0.192,0.631)$ & 0.293 \\
\hline Female tertile 3 & $0.134(-0.090,0.740)$ & 0.124 & $0.118(-0.127,0.701)$ & 0.173 & $0.136(-0.103,0.767)$ & 0.134 \\
\hline
\end{tabular}

Did not adjust for sex while analyzing in male or female PD patients, respectively. ASMI, appendicular skeletal muscle mass index; CI, confidence interval; PD, peritoneal dialysis; SUA, serum uric acid. ${ }^{\text {a }}$ Model 1 adjusted for age and gender. ${ }^{\mathrm{b}}$ Model 2 adjusted for model 1 covariables and diabetes, CVD, PD vintage, total Kt/V, use of active vitamin $\mathrm{D}_{3}$, uric acid-lowering agent, and diuretic. ${ }^{\mathrm{c}}$ Model 3 adjusted for model 2 covariables and neutrophil to lymphocyte ratio, serum albumin, triglyceride, and intact parathyroid hormone. ${ }^{\mathrm{d}}$ Reference group is the lowest tertile of SUA in total, male, and female patients, respectively.

remaining 147 male and 115 female middle-aged and older PD patients, among whom the mean SUA value was significantly higher in females than the males $(7.4 \pm 0.8$ vs. $6.2 \pm 0.9 \mathrm{mg} /$ $\mathrm{dL}, p<0.001$ ).

\section{SUA and All-Cause and CVD Mortality}

With a median follow-up of 68.7 (44.8-95.4) months, there were 4 patients $(0.50 \%)$ with recovering renal function, $138(17.2 \%)$ transferring to HD therapy, $178(22.2 \%)$ to renal transplantation, 2 patients $(0.25 \%)$ changing to other PD centers, and 3 patients $(0.37 \%)$ lost to follow-up. Moreover, 148 patients (18.5\%) died during the study period, including 73 (49.3\%) from cardiovascular events, 36 (24.3\%) from infection, 4 (2.7\%) from cancer, and 35 
Table 5. All-cause mortality for SUA in total, lower, and higher ASMI groups, respectively

\begin{tabular}{|c|c|c|c|c|c|c|}
\hline \multirow[t]{3}{*}{ Variables } & \multicolumn{6}{|l|}{ All-cause mortality } \\
\hline & \multicolumn{2}{|l|}{$\operatorname{model} 1^{\mathrm{a}}$} & \multicolumn{2}{|l|}{$\operatorname{model} 2^{\mathrm{b}}$} & \multicolumn{2}{|l|}{$\operatorname{model} 3^{c}$} \\
\hline & $\operatorname{HR}(95 \% \mathrm{CI})$ & $p$ value & HR $(95 \% \mathrm{CI})$ & $p$ value & HR $(95 \% \mathrm{CI})$ & $p$ value \\
\hline \multicolumn{7}{|l|}{ Continuous SUA } \\
\hline Total & $1.039(0.918,1.176)$ & 0.545 & $1.095(0.900,1.332)$ & 0.364 & $1.101(0.902,1.343)$ & 0.344 \\
\hline Lower ASMI group & $0.900(0.738,1.097)$ & 0.297 & $0.754(0.601,0.947)$ & 0.015 & $0.770(0.609,0.972)$ & 0.028 \\
\hline Higher ASMI group & $1.139(0.974,1.331)$ & 0.103 & $1.074(0.882,1.307)$ & 0.480 & $1.063(0.871,1.298)$ & 0.545 \\
\hline \multicolumn{7}{|l|}{ Categorical SUA ${ }^{\mathrm{d}}$} \\
\hline Total & $0.904(0.647,1.263)$ & 0.554 & $0.878(0.516,1.496)$ & 0.633 & $0.889(0.519,1.521)$ & 0.666 \\
\hline Lower ASMI group & $0.911(0.559,1.485)$ & 0.708 & $0.502(0.267,0.946)$ & 0.033 & $0.514(0.272,0.970)$ & 0.040 \\
\hline Higher ASMI group & $0.875(0.552,1.386)$ & 0.570 & $0.867(0.494,1.525)$ & 0.621 & $0.837(0.473,1.483)$ & 0.542 \\
\hline
\end{tabular}

Did not adjust for ASMI groups while analyzing in lower and higher ASMI PD patients, respectively. ASMI, appendicular skeletal muscle mass index; CI, confidence interval; ECW/TBW, extracellular water/total body water; PD, peritoneal dialysis; SUA, serum uric acid; HR, hazard ratio. $p$ value of the interaction between the ASMI and SUA groups, on all-cause mortality tested on the final model was 0.042. ${ }^{\text {a }}$ Model 1 adjusted for age and gender. ${ }^{b}$ Model 2 adjusted for model 1 covariables and Charlson comorbidity score, PD vintage, total Kt/V, use of angiotensin-converting enzyme inhibitor or angiotensin II receptor blocker, use of diuretic, use of uric acid-lowering agent, total cholesterol, high-density lipoprotein cholesterol, neutrophil to lymphocyte ratio, intact parathyroid hormone, ECW/TBW ratio $\geq 0.4$, ASMI groups, and ASMI groups $\times$ SUA. ${ }^{\mathrm{c}}$ Model 3 adjusted for model 2 covariables and serum albumin. ${ }^{\mathrm{d}}$ The hyperuricemia group versus the lower SUA group and reference group is the lower SUA group.

(23.6\%) from other causes (Fig. 1). There was a significant interaction between SUA and the different ASMI groups in all-cause mortality ( $p=0.042)$ but not in CVD mortality ( $p=0.118)$. By grouping PD patients according to median ASMI (shown in Table 5), the presence of hyperuricemia significantly predicted lower all-cause mortality (hazard ratio [HR] 0.514, 95\% CI $0.272,0.970 ; p=0.040$ ) in the lower ASMI group but not in the higher ASMI group (HR 0.837, $95 \%$ CI $0.473,1.483 ; p=0.542$ ). In addition, the adjusted HR of per $1 \mathrm{mg} / \mathrm{dL}$ higher SUA was $0.770(95 \%$ CI $0.609,0.972 ; p=0.028)$ for all-cause mortality only in lower ASMI patients.

\section{Discussion}

In this study, we characterized the relationship between SUA and ASM in PD patients. We demonstrated that elevated SUA levels were associated with greater ASM in middle-aged and older PD patients, and this positive association was significant in males but not in females, which was partly because of sex-related differences in SUA. Some studies have discussed the association between SUA and ASM. A previous large cross-sectional study including 3,079 participants reported that SUA was positively associated with ASMI in middle-aged and older "healthy" people using dual-energy X-ray absorptiometry [15]. Another study revealed a positive correlation between SUA and skeletal muscle index in postmenopausal women from Mexico [23]. The different sex-specific associations in PD patients revealed in our study may be explained by the following mechanism. First, males had significantly higher SUA levels than females $(7.0 \pm 1.4$ vs. $6.6 \pm 1.2 \mathrm{mg} / \mathrm{dL}$ in our study, $p<0.001)$. When males with SUA $>7.3$ $\mathrm{mg} / \mathrm{dL}$ or females with SUA $<6.3 \mathrm{mg} / \mathrm{dL}$ were excluded, the positive association was lost in males, suggesting there is a threshold value reached more frequently in males. Second, several studies demonstrated that estrogen promotes SUA secretion and leads to increases in SUA levels in postmenopausal women [24, 25]. Furthermore, estrogen deficiency was shown to 
cause accumulation of oxidative damage, which plays a vital role in skeletal muscle aging and loss [26]. Estrogen and its effects on premenopausal and postmenopausal females may partially weaken the significant association between SUA and ASM in the total female population. Third, males were shown to have greater age-related skeletal muscle mass decline, which may make the positive effect of SUA on ASM more readily observable [27, 28]. Overall, our results are consistent with the aforementioned studies. However, a large cross-sectional study from the Third National Health and Nutrition Examination Survey indicated that lower ASMI (defined as ASM/weight) measured by BIA was related to elevated SUA level [14]. Differences in the population analyzed, ASMI indices derived from height- or weight-adjusted models [27], and methods of measuring ASM [29] may partly explain the inconsistent observations.

The mechanisms underlying the positive association between ASMI and SUA are as follows. First, SUA was reported to be a nutritional marker in HD patients [3] and a similar observation was made in PD patients in our study (online suppl. Table 1). It is believed that higher SUA levels predict improved nutritional status in PD patients because of their specific diet rich in purine and fructose [30]. Furthermore, higher SUA levels were shown to correlate positively with the rate of normalized protein equivalent of nitrogen appearance [8]. Emerging data demonstrate that nutritional intervention with proteins was beneficial for skeletal muscle mass [31]. Therefore, it is believed that elevated SUA is associated with greater ASMI in PD patients because of their relatively better nutritional status. Second, increasing evidence indicates that the chemical milieu can cause UA to have antioxidant or prooxidant effects, which may partly explain the contradictory effects of UA in different studies [32]. As suggested intracellular UA has pro-oxidant effects [33, 34], while extracellularly it has antioxidant effects, serving as a powerful scavenger of free radicals [35,36]. Oxidative stress has been implicated as an essential factor in age-related loss of muscle mass, during which reactive oxygen species have been demonstrated to be overproduced [37] and cause oxidative damage to molecules including proteins, nucleic acids, and lipids. Moreover, the disruption of redox homeostasis during age-related loss of muscle mass modifies the activity of various transcription factors and kinases, such as FOXO, NF-kB, and MAPK family proteins, further inhibiting muscle regeneration [38]. Studies have shown that suppressing the redox process promotes both myoblast proliferation and differentiation $[39,40]$, which highlights the potential therapeutic effects of antioxidants in muscle aging and loss. The beneficial effects of UA as an antioxidant in bone health have been shown [41, 42], but there are few studies addressing the direct effects of UA on muscle health. Recently, an in vitro study found that exogenous UA increased oxidative stress in C2C12 skeletal muscle cells [43]. Therefore, the exact mechanism underlying the positive association between SUA and ASMI remains unclear, and additional studies are required. Regarding the difference between younger and older patients, this may be partially explained by the accelerated loss of muscle mass over time and the relatively poorer nutritional status of the older patients.

We demonstrated a significant interaction between the SUA and ASMI in all-cause mortality. After using the median ASMI to stratify patients, SUA was only found to be inversely associated with all-cause mortality in the lower ASMI group. Furthermore, the presence of hyperuricemia significantly predicted lower all-cause mortality in the lower ASMI group. Several studies have investigated the relationship between SUA and mortality in PD patients. Among them, in a retrospective study including 156 PD patients, Feng et al. [44] reported that higher SUA levels were associated with increased all-cause mortality. Xia et al. [12] reported that elevated SUA level was an independent risk factor for all-cause and CVD mortality in male PD patients [45] and CVD mortality in diabetic male PD patients [46] in a retrospective cohort study of 985 PD patients and 328 PD patients with diabetes, respectively. And, a study from Xiang et al. [12] showed that high SUA level was associated with higher all-cause but not CVD

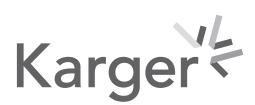


mortality, and the prognostic effects were more prominent in male, hypoalbuminemia, lower BMI, and nondiabetes patients subgroup [12]. Despite the direct association of SUA and mortality, some studies found no association between them. A retrospective study by Dong et al. [47] of 2,264 PD patients with follow-up of 26.5 months (median) revealed that the prognostic effects of SUA disappeared after adjusting for relevant risk factors. Similarly, studies by Bae et al. [48] and Li et al. [49] suggested there was no independent association. Recently, a study from Taiwan demonstrated that higher SUA levels were inversely associated with lower all-cause and CVD mortality in female PD patients but not in males [5]. Another study exploring the association between the longitudinal change of SUA and PD mortality showed that the decline of SUA during the follow-up period significantly predicted all-cause mortality [6]. In our study, a similar inverse association between SUA and all-cause mortality was identified, but only in the lower ASMI group. It appears the prognostic effects of SUA differ among those with different characteristics in the dialysis population. The reverse effects of SUA on PD mortality in the lower ASMI group in this study may be explained as follows. First, higher SUA level, which represents improved nutritional status, may exert positive effects on PD mortality more prominently in the lower ASMI population, which has relatively poorer nutrition. However, after adjusting for albumin levels, the reverse association remained statistically significant, indicating there may be other mechanisms that remain unidentified. Second, the beneficial antioxidant effects of SUA help improve prognosis in the lower ASMI population, which potentially has increased oxidative stress. Third, the risks associated with restricting foods rich in fructose and purine, or using UA-lowering agents to maintain SUA levels within the normal range, may exceed the harmful effects of higher SUA level itself. Fourth, the possibility of the "reverse epidemiology" effect, clearly demonstrated by Lai et al. [5], cannot be completely avoided. Therefore, it was hypothesized that the favorable effects of improved nutritional status or antioxidation may neutralize and even reverse the widely recognized adverse effects of higher SUA on mortality. However, whether higher SUA level acts as the cause or the effect of this favorable role remains to be determined.

Our study had some limitations including those inherent to single-center retrospective studies. Additionally, we lacked baseline SUA and BIA data. More convincing prospective or randomized controlled trials are required to further validate the relationship. We also lacked data on other nutritional markers analyzed extensively in PD patients, such as the Subjective Global Assessment Score and Malnutrition-Inflammation Score, which may help determine whether the inverse association between SUA and mortality is primarily because of nutritional effects. There are also potential unknown risk factors for mortality that may contribute to residual confounding effects. Finally, the BIA we used to measure body composition parameters is not the gold standard, although it is used extensively in PD patients [50]. Despite the aforementioned limitations, this was the first extensive study to evaluate the relationship between SUA and ASM and the effect of their interaction on mortality in PD patients. Moreover, since the relationship between ASM and SUA has primarily been investigated in older populations, there was a lack of knowledge of this relationship in younger patients. We enrolled a relatively large number of PD patients, including younger ones, to fully explore the relationship in representative population-based PD patient samples.

\section{Conclusion}

Our study revealed that elevated SUA levels were associated with greater ASMI in middleaged and older male PD patients, and independently predicted lower all-cause mortality in lower ASMI PD populations. Therefore, for PD populations with lower ASMI, clinicians are encouraged to assess the pros and cons of elevated SUA level and take nutritional status or

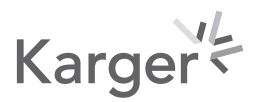


other relevant factors into consideration. Much more attention should also be paid to the lower SUA levels of PD patients. Additional prospective studies are required to further validate the effects of SUA on muscle health and determine the specific roles of SUA in different PD populations.

\section{Acknowledgements}

We thank all doctors and nurses in PD center for their meticulous work of patient care and data collection, and Richard Robins, PhD, from Liwen Bianji, Edanz Editing, China (www. liwenbianji.cn/ac), for editing the English text of a draft of the manuscript.

\section{Statement of Ethics}

All participants signed the informed consent. This study conforms to ethical standards of the Declaration of Helsinki and was approved by the Clinical Research Ethics Committee of the Sun Yat-sen University (Ethics approval number [2016] 215).

\section{Conflict of Interest Statement}

The authors declare no conflicts of interest.

\section{Funding Sources}

This work was supported by the Natural Science Foundation of China (Grant Nos. 81774069, 81570614), National Key Research and Development Program (Grant No. 2016YFC0906101), Foundation of Guangdong Key Laboratory of Nephrology (Grant No. 2017B030314019), the Guangdong Science Foundation of China (Grant No. 2017A050503003, 2017B020227006), and the Guangzhou Committee of Science and Technology, China (201704020167).

\section{Author Contributions}

X. Yu and X.X. designed the research; C.Y. and X.X. conducted the research; Y.P., M.W., and X.H. collected data; H.W., H.Y., and C.Y. analyzed the data; X. Yu and X. Yang interpreted the findings; X.X. wrote the paper; and X. Yang had the primary responsibility for the whole content. All authors read and approved the final version of the manuscript.

\section{References}

1 von Lueder TG, Girerd N, Atar D, Agewall S, Lamiral Z, Kanbay M, et al. Serum uric acid is associated with mortality and heart failure hospitalizations in patients with complicated myocardial infarction: findings from the High-Risk Myocardial Infarction Database Initiative. Eur J Heart Fail. 2015 Nov;17(11):1144-51.

2 Cheong E, Ryu S, Lee JY, Lee SH, Sung JW, Cho DS, et al. Association between serum uric acid and cardiovascular mortality and all-cause mortality: a cohort study. J Hypertens. 2017 May;35(Suppl 1):S3-9.

3 de Jager DJ, Grootendorst DC, Jager KJ, van Dijk PC, Tomas LM, Ansell D, et al. Cardiovascular and noncardiovascular mortality among patients starting dialysis. JAMA. 2009 Oct;302(16):1782-9. 
4 Murea M, Tucker BM. The physiology of uric acid and the impact of end-stage kidney disease and dialysis. Semin Dial. 2019 Jan;32(1):47-57.

5 Lai KJ, Kor CT, Hsieh YP. An inverse relationship between hyperuricemia and mortality in patients undergoing continuous ambulatory peritoneal dialysis. J Clin Med. 2018 Nov; 7(11):e416.

6 Chang W, Uchida S, Qi P, Zhang W, Wang X, Liu Y, et al. Decline in serum uric acid predicts higher risk for mortality in peritoneal dialysis patients: a propensity score analysis. J Nephrol. 2020 Jun;33(3):591-9.

7 Strazzullo P, Puig JG. Uric acid and oxidative stress: relative impact on cardiovascular risk? Nutr Metab Cardiovasc Dis. 2007 Jul;17(6):409-14.

8 Beberashvili I, Sinuani I, Azar A, Shapiro G, Feldman L, Stav K, et al. Serum uric acid as a clinically useful nutritional marker and predictor of outcome in maintenance hemodialysis patients. Nutrition. 2015 Jan;31(1): 138-47.

9 Fouque D, Kalantar-Zadeh K, Kopple J, Cano N, Chauveau P, Cuppari L, et al. A proposed nomenclature and diagnostic criteria for protein-energy wasting in acute and chronic kidney disease. Kidney Int. 2008 Feb; 73(4):391-8.

10 Jin S, Lu Q, Su C, Pang D, Wang T. Shortage of appendicular skeletal muscle is an independent risk factor for mortality in peritoneal dialysis patients. Perit Dial Int. 2017;37(1):78-84.

11 Kang SH, Cho KH, Park JW, Do JY. Low appendicular muscle mass is associated with mortality in peritoneal dialysis patients: a single-center cohort study. Eur J Clin Nutr. 2017 Dec;71(12):1405-10.

12 Xiang S, Zhang X, Xie X, Wang J, Zhou Q, Chen Z, et al. High serum uric acid level is a mortality risk factor in peritoneal dialysis patients: a retrospective cohort study. Nutr Metab. 2019 Aug;16:52.

13 Faulkner JA, Larkin LM, Claflin DR, Brooks SV. Age-related changes in the structure and function of skeletal muscles. Clin Exp Pharmacol Physiol. 2007 Nov;34(11):1091-6.

14 Beavers KM, Beavers DP, Serra MC, Bowden RG, Wilson RL. Low relative skeletal muscle mass indicative of sarcopenia is associated with elevations in serum uric acid levels: findings from NHANES III. J Nutr Health Aging. 2009 Mar;13(3):177-82.

15 Dong XW, Tian HY, He J, Wang C, Qiu R, Chen YM. Elevated serum uric acid is associated with greater bone mineral density and skeletal muscle mass in middle-aged and older adults. PLoS One. 2016 May; 11(5): e0154692.

16 Huang R, Liu Y, Wu H, Guo Q, Yi C, Lin J, et al. Lower plasma visceral protein concentrations are independently associated with higher mortality in patients on peritoneal dialysis. Br J Nutr. 2015 Feb;113(4):627-33.

17 Charlson ME, Pompei P, Ales KL, MacKenzie CR. A new method of classifying prognostic comorbidity in longitudinal studies: development and validation. J Chronic Dis. 1987;40(5):373-83.

18 Guo Q, Yi C, Li J, Wu X, Yang X, Yu X. Prevalence and risk factors of fluid overload in Southern Chinese continuous ambulatory peritoneal dialysis patients. PLoS One. 2013;8(1):e53294.

19 Chen LK, Liu LK, Woo J, Assantachai P, Auyeung TW, Bahyah KS, et al. Sarcopenia in Asia: consensus report of the Asian working group for sarcopenia. J Am Med Dir Assoc. 2014 Feb;15(2):95-101.

20 Kastner M, Wilczynski NL, Walker-Dilks C, McKibbon KA, Haynes B. Age-specific search strategies for Medline. J Med Internet Res. 2006 Oct;8(4):e25.

21 Yamanaka H; Japanese Society of Gout and Nucleic Acid Metabolism. Japanese guideline for the management of hyperuricemia and gout: second edition. Nucleosides Nucleotides Nucleic Acids. 2011 Dec;30(12):1018-29.

22 Ko MJ, Yun S, Oh K, Kim K. Relation of serum 25-hydroxyvitamin D status with skeletal muscle mass by sex and age group among Korean adults. Br J Nutr. 2015 Dec;114(11):1838-44.

23 Zacarias-Flores M, Sanchez-Rodriguez MA, Garcia-Anaya OD, Correa-Munoz E, Mendoza-Nunez VM. Relationship between oxidative stress and muscle mass loss in early postmenopause: an exploratory study. Endocrinol Diabetes Nutr. 2018 Jun-Jul;65(6):328-34.

24 Sumino H, Ichikawa S, Kanda T, Nakamura T, Sakamaki T. Reduction of serum uric acid by hormone replacement therapy in postmenopausal women with hyperuricaemia. Lancet. 1999 Aug;354(9179):650.

25 Hak AE, Choi HK. Menopause, postmenopausal hormone use and serum uric acid levels in US women-the third national health and nutrition examination survey. Arthritis Res Ther. 2008;10(5):R116.

26 Buonocore D, Rucci S, Vandoni M, Negro M, Marzatico F. Oxidative system in aged skeletal muscle. Muscles Ligaments Tendons J. 2012 Feb;1(3):85-90.

27 Kim KM, Jang HC, Lim S. Differences among skeletal muscle mass indices derived from height-, weight-, and body mass index-adjusted models in assessing sarcopenia. Korean J Intern Med. 2016 Jul;31(4):643-50.

28 Oh SL, Yoon SH, Lim JY. Age- and sex-related differences in myosin heavy chain isoforms and muscle strength, function, and quality: a cross sectional study. J Exerc Nutrition Biochem. 2018 Jun;22(2):43-50.

29 Tewari N, Awad S, Macdonald IA, Lobo DN. A comparison of three methods to assess body composition. Nutrition. 2018 Mar; 47:1-5.

30 Choi HK, Liu S, Curhan G. Intake of purine-rich foods, protein, and dairy products and relationship to serum levels of uric acid: the third national health and nutrition examination survey. Arthritis Rheum. 2005 Jan; 52(1):283-9.

31 Forbes SC, Little JP, Candow DG. Exercise and nutritional interventions for improving aging muscle health. Endocrine. 2012 Aug;42(1):29-38.

32 Sautin YY, Johnson RJ. Uric acid: the oxidant-antioxidant paradox. Nucleosides Nucleotides Nucleic Acids. 2008 Jun;27(6):608-19. 
33 Sautin YY, Nakagawa T, Zharikov S, Johnson RJ. Adverse effects of the classic antioxidant uric acid in adipocytes: NADPH oxidase-mediated oxidative/nitrosative stress. Am J Physiol Cell Physiol. 2007 Aug;293(2): C584-96.

34 Yu MA, Sánchez-Lozada LG, Johnson RJ, Kang DH. Oxidative stress with an activation of the renin-angiotensin system in human vascular endothelial cells as a novel mechanism of uric acid-induced endothelial dysfunction. J Hypertens. 2010 Jun;28(6):1234-42.

35 Ames BN, Cathcart R, Schwiers E, Hochstein P. Uric acid provides an antioxidant defense in humans against oxidant- and radical-caused aging and cancer: a hypothesis. Proc Natl Acad Sci U S A. 1981 Nov;78(11):685862.

36 Glantzounis GK, Tsimoyiannis EC, Kappas AM, Galaris DA. Uric acid and oxidative stress. Curr Pharm Des. 2005;11(32):4145-51.

37 Sakellariou GK, Jackson MJ, Vasilaki A. Redefining the major contributors to superoxide production in contracting skeletal muscle. The role of NAD(P)H oxidases. Free Radic Res. 2014 Jan;48(1):12-29.

38 Meng SJ, Yu LJ. Oxidative stress, molecular inflammation and sarcopenia. Int J Mol Sci. 2010Apr;11(4):1509_ 26.

39 Hansen JM, Klass M, Harris C, Csete M. A reducing redox environment promotes C2C12 myogenesis: implications for regeneration in aged muscle. Cell Biol Int. 2007 Jun;31(6):546-53.

40 Derbre F, Gratas-Delamarche A, Gomez-Cabrera MC, Vina J. Inactivity-induced oxidative stress: a central role in age-related sarcopenia? Eur J Sport Sci. 2014;14(Suppl 1):S98-108.

41 Ahn SH, Lee SH, Kim BJ, Lim KH, Bae SJ, Kim EH, et al. Higher serum uric acid is associated with higher bone mass, lower bone turnover, and lower prevalence of vertebral fracture in healthy postmenopausal women. Osteoporos Int. 2013 Dec;24(12):2961-70.

42 Li HZ, Chen Z, Hou CL, Tang YX, Wang F, Fu QG. Uric acid promotes osteogenic differentiation and inhibits adipogenic differentiation of human bone mesenchymal stem cells. J Biochem Mol Toxicol. 2015 Aug;29(8): 382-7.

43 Maarman GJ, Andrew BM, Blackhurst DM, Ojuka EO. Melatonin protects against uric acid-induced mitochondrial dysfunction, oxidative stress, and triglyceride accumulation in C2C12 myotubes. J Appl Physiol. 2017 Apr;122(4):1003-10.

44 Feng S, Jiang L, Shi Y, Shen H, Shi X, Jin D, et al. Uric acid levels and all-cause mortality in peritoneal dialysis patients. Kidney Blood Press Res. 2013;37(2-3):181-9.

45 Xia X, He F, Wu X, Peng F, Huang F, Yu X. Relationship between serum uric acid and all-cause and cardiovascular mortality in patients treated with peritoneal dialysis. Am J Kidney Dis. 2014 Aug;64(2):257-64.

46 Xia X, Zhao C, Peng FF, Luo QM, Zhou Q, Lin ZC, et al. Serum uric acid predicts cardiovascular mortality in male peritoneal dialysis patients with diabetes. Nutr Metab Cardiovasc Dis. 2016 Jan;26(1):20-6.

47 Dong J, Han QF, Zhu TY, Ren YP, Chen JH, Zhao HP, et al. The associations of uric acid, cardiovascular and allcause mortality in peritoneal dialysis patients. PLoS One. 2014 Jan;9(1):e82342.

48 Bae E, Cho HJ, Shin N, Kim SM, Yang SH, Kim DK, et al. Lower serum uric acid level predicts mortality in dialysis patients. Medicine (Baltimore). 2016 Jun;95(24):e3701.

49 Li W, Xiong L, Fan L, Wang Y, Peng X, Rong R, et al. Association of baseline, longitudinal serum high-sensitive C-reactive protein and its change with mortality in peritoneal dialysis patients. BMC Nephrol. 2017 Jul;18(1): 211.

50 Medici G, Mussi C, Fantuzzi AL, Malavolti M, Albertazzi A, Bedogni G. Accuracy of eight-polar bioelectrical impedance analysis for the assessment of total and appendicular body composition in peritoneal dialysis patients. Eur J Clin Nutr. 2005 Aug;59(8):932-7. 